tionären Regierung und der im Volk verankerten katholischen Kirche. So ist auch heute noch die Gegnerschaft zwischen Kirche und Gewerkschaften jederzeit virulent: Die Behauptung eines Gewerkschaftsführers etwa, der Erzbischof von Cuernavaca unterhalte Guerillalager ${ }^{7}$, erinnert zwangsläufig auch an die hier behandelte jüngste Vergangenheit.

Im II. Teil seiner Arbeit (S. 67-200) gibt M. einen Einblick in die soziale und geographische Herkunft der Cristeros und ihrer Basis, schildert detailliert (!) und doch jederzeit verständlich Organisation, Finanzierung, Bewaffung und Logistik der Rebellenarmee sowie Zusammensetzung und Funktion der Führung. Abschließend (Teil III) werden die Auswirkungen der Rebellion auf Staat, Kirche, Cristero-Bewegung 8 und die mexikanische Revolution dargestellt. Die Bibliographie weist das umfängliche Quellenmaterial sowie die Sekundärliteratur gegliedert nach Herkunft und Gegenstand nach.

Gerhard Scheffler, Hamburg

\title{
LUdGER REUke
}

\section{Befreier und Erlöser? Militär und Entwicklung in Ghana Bonn 1976, 215 S. (Verlag Neue Gesellschaft)}

Das Ghana nach Nkrumah hat weit weniger wissenschaftliche Beachtung und Würdigung gefunden als das Land des Osagyefo, mit dem quasi der ganze Kontinent begann, in die völkerrechtliche Unabhängigkeit aufzubrechen. Um so erfreulicher ist es, die Arbeit von Ludger Reuke vorzustellen, die im Rahmen einer Arbeitsgruppe „Militär und Entwicklung in der Dritten Welt" entstanden ist und dem Verfasser 1971/72 und später noch einmal 1974 und 1975 zur Materialsammlung nach Ghana geführt hat, wo er u. a. eine Reihe von Interviews mit führenden ghanaischen Politikern und Militärs geführt hat. Den beiden von ihm untersuchten Militärregimen, dem NLC (1966-69) und dem NRC (seit 1972, bei Reuke bis 1975), denen sein eigentliches Interesse gilt, stellt er zwei Kapitel über die Entwicklung der ghanaischen Gesellschaft bzw. die Entwicklung der zivil-militärischen Beziehungen bis zum Sturz Nkrumahs bzw. unter Busia voran. In diesen versucht er den Fragen nachzugehen, inwiefern die gesamtgesellschaftliche Entwicklung (so wie sie von den zivilen Regimen gestaltet wurde) die Gelegenheit zur Intervention der Militärs schuf bzw. bei diesen auch den Willen zur Intervention heranreifen ließ. Die Militärregime untersucht er dann in jeweils zwei Hauptkapiteln unter den Fragestellungen „Etablierung des Regimes“, mit den Unterkapiteln „Rechtsertigung und Zielsetzung“, „erste Maßnahmen“, „der Entscheidungsprozeß“, und „Die entwicklungsrelevante Politik des Regimes“, mit den Unterabschnitten „Die allgemeinen Voraussetzungen zu einer Entwicklung“, „Der NLC/NRC als Machtelite“, „Die politische Elite“ sowie die „Wirtschafts- und Sozialpolitik“ bzw. die "Außenpolitik“. Ihm ist damit eine gut lesbare, flüssig geschriebene, überwiegend kompetent recherchierte, mit einem auch in Einzelfragen ausgewogenen Urteil versehene Arbeit gelungen, die auch straff genug geschrieben ist, um lesbar zu sein.

\footnotetext{
7 Domitra, M: „Die Rolle der Gewerkschaften im mexikanischen Herrschaftssystem“, Bonn-Bad Godesberg 1975 , S. $243 \mathrm{f}$.

8 Gewisse Verbindungen zur faschistoiden Sinarquistenbewegung der dreißiger Jahre sind nicht zu leugnen, S. $212 \mathrm{ff}$., vgl. de la Vega-Leinert, A. M.: "El sinarquismo en México: posibilidades de un régimen fascista en $1940^{\alpha}$, in: Comercio Exterior 1976, S. $1076 \mathrm{ff}$.
} 
Dennoch müssen einige Fragezeichen in methodischer und theoretischer Hinsicht sowie in der Interpretation einzelner Probleme gesetzt werden: Reuke geht von zwei zentralen Thesen aus, von denen die eine zu enge Perspektive, eine nur partielle Gültigkeit hat (und haben kann), deren andere zwar richtig ist, methodisch aber nur partiell befriedigend angegangen wird. Zunächst zur letzteren: Nach Reuke ist eine zielgerichtete Entwicklung aller Bereiche, die auf menschliche Arbeit angewiesen sind, theoretisch nur aufgrund von $Z$ wangsmaßnahmen oder aufgrund von Konsensus möglich. Wegen der völlig unzureichenden Infrastruktur, der geringen Stärke, Differenzierung und Ausbreitung von Bürokratie, Polizei und Armee, scheide die Entwicklung aufgrund von Zwangsmaßnahmen in Afrika südlich der Sahara im allgemeinen und auch in Ghana im besonderen aus. Demnach wäre hier der Konsensus eine unabdingbare Voraussetzung für Entwicklung (S. 108). Der Rezensent kann dem nicht widersprechen. Allerdings muß dieser Konsensus nicht nur auf die verschiedenen Fraktionen der politischen, sozialen und ökonomischen Eliten sich erstrecken, sondern namentlich auch die Beziehungen der Eliten zu den Massen bzw. zwischen herrschender Klasse und den abhängigen Klassen, miteinbeziehen. Gerade die letzteren Gruppen erfaßt Reuke mit seiner Arbeit aber nicht oder nur am Rande bzw. spekulativ. Er untersucht und berücksichtigt im wesentlichen nur die Reaktionen von Elitegruppen und -personen, die sich nachweisbar („im nationalen Rahmen“) artikulieren können. Damit wird sicherlich nicht immer nur der genannte Personenkreis selbst erfaßt, sondern auch größere gesellschaftliche Gruppen (oder wenigstens relevanter Teile davon), die durch diese repräsentiert werden (Intelligentsia, Beamtenschaft, Arbeiterklasse usw.). In Gesellschaften, die aber noch so rudimentär gesellschaftlich formiert sind wie Ghana, werden hier aber große Teile der Bevölkerung übersehen, auf die es bei einer Entwicklungsmobilisierung gerade ankommen würde: die Massen mit niederem ökonomischen und sozialen Status, die villeicht nur marginal in die moderne OKonomie einbezogen sind, denen keine genuinen gesellschaftlichen und politischen Organisationen zur Verfügung stehen, deren politischer Dissenz mit den Machthabern daher notwendigerweise a-politisch sein muß und durch äußere Passivität, „Apathie“, innere Emigration ausgedrückt wird, und die sich nicht artikulieren durch diese oder jene Wahlentscheidung, Beteiligung an Streiks und Demonstrationen, Attentaten, Putschversuchen, politischen Kommentaren und Erklärungen (die von den Medien zur Kenntnis genommen und $u$. U. verbreitet werden). Dies trifft in erster Linie (aber nicht nur) auf die Landbevölkerung, insbesondere auf die quasi Subsistenzbauern, zu. Als ein (allein nicht ausreichender) Indikator dafür kann die Wahlbeteiligung angesehen werden. Diese betrug so z. B. 1969 in der Nordregion nur $37 \%$ gegenüber $70,5 \%$ in Accra ${ }^{1}$. Um diesen Konsens zwischen Herrschern und Beherrschten und die Legitimität der Herrschenden bzw. die Loyalität der Beherrschten ermitteln zu können, bedarf es der Mikrostudien. Ein Gebiet, an das sich Politologen in Deutschland (anders als etwa in Amerika), wohl aus den verschiedensten Gründen, noch nicht heranwagen, obwohl die Notwendigkeit, aufgrund der entwicklungstheoretischen und staatstheoretischen Diskussion, immer evidenter wird. Reuke wäre durch die hier angesprochene Ausweitung der Methode möglicherweise

\footnotetext{
1 Vgl. Tetzlaff, R.: in Ansprenger, F. u. a.: Die politische Entwicklung Ghanas von Nkrumah bis Busia, München 1972, S. 197.
} 
(zeitlich) überfordert gewesen. Auf die Grenzen seiner Methode hätte er aber hinweisen können. Ansonsten breitet er Dissens und Konsens auf Eliten- und „nationaler“ Ebene im allgemeinen durchaus kompetent, mit vielen bisher unbekannten Einzelheiten, aus, jedenfalls was die Zeit nach 1966 betrifft. Für die Nkrumah-Zeit trägt er hingegen einige z. T. in der Literatur gängige Fehleinschätzungen weiter: So ist z. B. nicht einsichtig, warum es ausgerechnet in den frühen 50er Jahren einen "Grundkonsensus" zwischen allen am politischen Prozeß beteiligten Personen und Gruppen gegeben haben soll (S. 23, S. 198), der später verlorenging, wo die CPP und die jeweilige Opposition sich nicht einmal einig über die Unabhängigkeitswerdung und die zukünftige verfassungsrechtliche Form waren und diese Auseinandersetzungen durchaus gewaltsame Formen annahm (deren Verantwortung Reuke wohl etwas unberechtigt der CPP anlastet, S. 31). Andererseits stimmt es auch nicht, daß nach 1962 die innerparteiliche Kritik erstarb (S. 38, implizit, S. 124). Von dem Kampf der „socialist boys“ im Spark gegen das CPP-Establishment einmal ganz abgesehen wurde das Parlament, nach (zwangsweisem) Ubertritt der letzten Oppositionsabgeordneten, eher noch lebendiger als zuvor. Kritik wurde und konnte zwar nicht an Nkrumah und dem Nkrumaismus (was keiner so recht wußte, was das war) noch an der Außenpolitik geübt werden, dafür aber um so mehr an der Wirtschaftspolitik, einzelnen Verwaltungsmaßnahmen und der fehlenden persönlichen Integrität von (hohen) Amtsinhabern. Noch im August 1965 konnte so z. B. der Abgeordnete Iddrissu verkünden: „Those who get rich quick are none else but those who are around the President" ${ }^{\text {"2 }}$ Erst als das Parlament auf Betreiben desselben Abgeordneten gegen den erbitterten Widerstand der Regierung in einer tumultuarischen Sitzung eine Resolution verabschiedete, in der die Regierung und das ZK (der Partei) aufgefordert wurde, die Ziele und Absichten des "Dawn Broadcast" des Presidenten (von 1961!, in dem er u. a. gegen die Korruption gesprochen hatte) zu verwirklichen, wurde dieses (auch öffentlichkeitswirksame) Forum der Diskussion, der Kritik und des Dissenses durch den Entzug des Mandates von Indrissu, der wenige Tage vor dem Coup erfolgte, mundtot gemacht.

Wichtiger als diese Einzelkritiken ist aber vielleicht noch ein anderer Punkt: Reuke sieht "das zentrale Problem Ghanas" nicht in der Wirtschaft, sondern im politisch institutionellen Bereich, ja darüber hinaus im Wertsystem" (S. 127, S. 202). Darunter versteht er insbesondere Wertvorstellungen, „die einerseits bei den Regierten eine überzogene Heilserwartung hervorrufen und andererseits die Regierenden den Staat als Beute erscheinen lassen ". Daß es sich hierbei um einen sehr wichtigen, möglicherweise zentral wichtigen Punkt handelt, vermag Reuke durchaus überzeugend darzulegen. Das genannte Problem aber als den zentralen Punkt auszugeben und die Analyse dementsprechend einzuschränken, scheint für die meisten "peripheren" Gesellschaftsformationen und ihre Staatsapparate im allgemeinen und für Ghana im besonderen doch sehr fragwürdig. Sicherlich ist dieser Aspekt - ungerechtfertigterweise - durch Dependenz- und Imperialismusanalytiker und -theoretiker (wohl auch als Reaktion auf die "endogenen" Faktoren nahezu ausschließlich behandelnde [ältere] amerikanische Modernisierungstheorie) etwas zu stark in den Hintergrund gedrückt worden und Entwicklung und Unterent-

2 Dazu: Hanisch, Rolf: Der Handlungsspielraum eines Landes der Peripherie im internationalen System. Das Beispiel Ghanas. Saarbrücken 1975, S. 424, für den gesamten Komplex: 420-426. 
wicklung zu einseitig aus der Perspektive der internationalen Dominanz- und Dependenzverhältnisse gesehen worden. Es soll hier auch nicht gestritten werden, welcher Aspekt wichtiger ist, was sicherlich auch im Einzelfall entschieden werden müßte. Von vornherein ausgeklammert sollte dieses Problem jedoch nicht werden. So kann man Ziele und Strategien (oft auch nicht die Genese) eines Regimes nicht verstehen, wenn man nicht auch auf seine (aktuelle und potentielle) Ressourcenbasis eingeht, die wesentlich mit von den intra-nationalen Determinanten bestimmt wird. So kann die aktuelle Ressourcenbasis eines Regimes von außen (auch kurzfristig) eingeschränkt oder auch erweitert werden. Angesichts der erheblichen Abhängigkeit der Gesamtwirtschaft, der Zahlungsbilanz, des staatlichen Budgets etwa von der Weltmarktentwicklung einiger Rohstoffe, die exportiert werden, können die Mittel für Konsens schaffende soziale Befriedigungsstrategien so entweder vorhanden sein oder bereitgestellt werden oder auch so eingeschränkt werden, daß Austerity-Maßnahmen notwendig werden, die bei den Betroffenen leicht ein Dissens-Potential ausweiten oder erst schaffen. Es ist offensichtlich, daß ein korruptes, im ganzen ineffizientes Regime diesen (positiven) Handlungsspielraum $z$. T. vergeuden und nicht so zielmitteladequat einsetzen kann wie etwa ein nichtkorruptes, effizienter wirtschaftendes Regime, das sich vielleicht auch besser über außenwirtschaftliche Engpässe und Krisen hinwegzuhelfen vermag. Es ist auch vorstellbar, daß ein Regime (mehr als ein anderes) seine Ressourcenund Legitimitätsbasis durch Aktionen im intern. System zu erweitern (u. U. auch mehr oder weniger allein darauf zu gründen) sucht. In jedem Fall sind die Interaktionen zwischen nationalem und internationalem System, zwischen Autonomie und intern. Abhängigkeit und Eingrenzung, zwischen Eigenverantwortlichkeit und Fremdbestimmung, eine zentral wichtige Fragestellung.

Es ist nicht so, daß Reuke diesen Aspekt überhaupt nicht sieht. In der Schlußbetrachtung bemerkt er, daß das "Grundübel der ghanaischen Wirtschaft" die „übergroße Abhängigkeit vom Kakao“, von keinem der untersuchten Regime entscheidend abgeholfen worden sei (S. 200). Auch erwähnt er den positiven Effekt der. steigenden Kakaopreise für die jeweiligen Militärregime (S. 133 und 191), den negativen Effekt der fallenden Preise für die Nkrumah-Endphase (S. 44, nicht jedoch für Busia!). Dies geschieht jedoch mehr beiläufig, als zentrale Kategorie werden die Weltmarktpreisschwankungen für Kakao (neben anderen Indikatoren äußerer Abhängigkeit bzw. anderen transnationalen Interaktionen) nicht eingeführt. So vermißt man auch nahezu jeglichen Hinweis auf eine Bewertung der noch sehr engen Beziehungen des NLC zum IWF, zur Weltbank und zu den USA, auf den Einfluß der permanenten IWF-Mission, ferner der sog. „Harvard Advisory Group“, auf die Wirtschaftsplitik des NLC. Wir denken da u. a. an die Formulierung des ersten Budgets, die Abwertung 1967 („Ghana entschloß sich hier" durchaus nicht, S. 131), an das Gerangel um die Freigabe der seit Jahren faktisch eingefrorenen zu retransferierenden Gewinne, aber auch an die Scheinerfolge, die Ghana durch diese engen Bindungen zu erzielen vermochte: Unterstützung bei den Umschuldungen der privaten Lieferantenkredite mit vor allem europ. Ländern, Abschluß einer Úbereinkunft mit den USA über Grundprinzipien eines internationalen Kakaoabkommens (1967), das sich dann aber nicht materialisierte.

Hochinteressant ist sonst Reuke immer dort, wo er die Entscheidungs- und Willensbildungsprozesse der Militärregime als "ghanaische Elite“ nachzuzeichnen versucht. Er trägt so auch eine Reihe von eindrucksvollen Belegen für seine Thesen 
zusammen, daß die Militärs aufgrund ihres Machtmonopols eher in der Lage seien, kurzfristig wichtige Entscheidungen durchzusetzen als Zivilregime, daß sie bei langfristigen Aufgaben jedoch normalerweise nicht die notwendige Einsicht in die Komplexität der Entscheidung hätten, die zu einem längerfristigen Erfolg führen könnten (S. 126). Viele seiner Entscheidungen mußte so der NLC sehr bald wieder modifizieren bzw. wieder aufheben, während der NRC, der durchaus nicht seltener vor so mißlichen Entscheidungen steht, eher dazu neigt, den ursprünglichen Beschluß trotz alledem durchzuziehen. Überhaupt trägt Reuke überzeugendes Material über den NRC zusammen, das geeignet ist, die ursprüngliche Euphorie von einigen Dependenztheoretikern, immer auf der Suche nach "autozentrierten“ Entwicklungsstrategien in der Dritten Welt, die hier auch schon eine genuine Politik der „self-reliance“ wahrnehmen wollten ${ }^{3}$, ganz erheblich zu dämpfen. Reuke macht deutlich, daß neben korporativen besonders auch persönliche Interessen zum erfolgreichen Putsch geführt haben (S. 159), daß jegliche Gedanken über eine Politik nach dem Putsch fehlten, die sich dann - im positiven wie im negativen - als eine bloße Umkehr der bisherigen Politik darstellte, ohne jedoch „sozialistisch“ zu werden. Trotz der kritischen Einwände, eine sehr lesenswerte Arbeit.

Rolf Hanisch

\section{comité information sahel}

qui se nourrit de la famine en afrique? le dossier politique de la faim au sahel cahiers libres 292-293, françois maspero, Paris 1974, 278 S.

Deutschsprachige Berichte zur Dürrekatastrophe in den sechs Sahelländern deckten bei einer Erklärung ihrer Ursachen hauptsächlich technische Mängel wie Wassermangel, übermäßiges Abweiden und Abbrennen und die damit verbundenen Bodenerosionen auf, Faktoren, die zur Desertifikation weiter Landstriche führten. Die wenigen Kritiken entwicklungspolitischer Art beschränkten sich meistens (mangels genauerer Kenntnisse dieser frankophonen Länder?) auf recht allgemeine Merkmale der Unterentwicklung.

Ahnlich einseitig verlief die Berichterstattung in Frankreich, dem ehemaligen „Mutterland“ der sechs dürregeschädigten Sahelländer. Nachdem sich das Schweigen der französischen Regierung (im Mai 1973 fanden die Präsidentschaftswahlen statt), der betroffenen afrikanischen Staatschefs, einiger etablierter Afrikanisten sowie der Presse zu den politischen Hintergründen der Krise weitgehend als hartnäckige Interessenpolitik herausgestellt hatte, veröffentlichte eine Gruppe von 86 französischen Afrika-Forschern den vorliegenden Bericht.

Ziel dieser Studie ist es, die über die Merkmale einer reinen Naturkatastrophe hinausgehenden ursächlichen entwicklungspolitischen und -ökonomischen Bedingungsfaktoren aufzudecken, um damit die Sahel-Situation aus ihrem gesamtgesellschaftlichen Kontext heraus besser erklären zu können. Die Autoren erbringen dabei den unbequemen Nachweis, daß die Ursache für die Hungerkatastrophe nicht allein die Trockenheit - ein gewohntes, in unregelmäßigen Abständen auftretendes Phänomen - sein konnte, sondern in einer jahrzehntelangen Mono-

\footnotetext{
3 Vgl. Tetzlaff, R.: Ghana - Fehlgeschlagene Versuche der Befreiung, in: Grohs, G./Tibi, B. (Hrsg.):
} Zur Soziologie der Dekolonisation in Afrika, Frankfurt 1973, S. 261/262. 